\title{
On McStay's Emotional AI: Beyond the basic emotions
}

\author{
Ho Manh Tung \\ Ritsumeikan Asia Pacific University \\ Beppu, Oita, Japan
}

October 22, 2020

In Emotional AI: The rise of empathic media, McStay (2018) presents one of the complete accounts of the current state-of-the-art emotional artificial intelligence (AI) systems. These are, according to the author, the new media technologies that are acquiring greater ability to read, sense, track, and classify human emotions, thanks to the rise of big data and computational power. The author calls our attention to a plethora of new concepts such as empathic media, empathic cities, or algocracy, which are clearly relevant to our $21^{\text {st }}$ century being, yet they remain largely esoteric to the public.

I have discussed in some details McStay (2018)'s account of empathic media can benefit from further differentiation of the nuances in the concept empathy (Ho, 2020). In this essay, I discuss different theories of emotion and how they might influence the quantification and datafication of emotions, which have serious implications for the premise of the book.

McStay states: "Empathic media employ a particular account of what emotions are through their use of psychological, anthropological, and neuroscientific research, largely deriving from Paul Ekman and his forerunners" (2018, p.4). Later on, he further elucidates that this account of emotion is based on the assumption of "basic emotions" that are championed by Paul Ekman (1983, 
1999). Logically, this view that emotions are basic and readily recognizable across cultures is very attractive to the large data-mining and AI industry. First, buying into this premise means that as long as we have enough data, we will be able to train our algorithms to correctly identify emotions. And second, as tracking emotions enables predicting behaviors, emotional AI systems and empathic media are of a great interest for all organizations whether for commercial, political, or scientific purposes.

McStay only briefly mentions the social constructionist, ethnocentric, context-dependent views of emotions in the book, stating that due to its complexity, the tech and business communities have not picked up on it. In my opinion, this treatment of the alternative theories of emotion will present significant limitations to any discussion of the ethics, governance, and accuracy of emotional AI. He states "While proponents of categorical approaches (to emotions) do not fully answer Russell's (1994) ethnocentric criticisms, they embrace measurable dimensional factors to triangulate and strengthen conclusions about the extent to which a person is undergoing a named emotion" (McStay, 2018, p.60). This again echoes the view of "machinic verisimilitude," (p. 4), which states we can leave aside the issue of authenticity as long as machines produce a good enough accuracy.

It is true that Ekman's basic emotions are completely aligned with the computational interest in categorizing and passively detecting emotions, but its utility and simplicity should not, first and foremost, lead us to believe our emotional lives only consist of the eight basic emotions (joy, surprise, sadness, contempt, anger, fear, disgust, and trust) and the three overarching sentiments (negative, neutral, positive). Furthermore, this impoverished and reductionist view of emotions should worry us because our decision-making relies more on more on these technologies 
since they are increasingly deployed to surveil public spaces, to monitor public sentiments on social media, to customize our news feed.

To illustrate the poverty of this view and its technical implications. First, even if there are really only eight basic emotions, each motion is accompanied with many shades or granularities (Barrett, 2017). For example, anger can come into the shade of hostility, aggregation, frustration, etc. Thus, at the very least, I believe, some discussions on how to expand on the "basic emotions paradigm" must be presented. For instances, we can add to the following categories to Ekman's list: awe, curiosity, doubt, confusion, shame, pride, stress, embarrassment, indifference. It seems to me given the decline of computational cost, the cost of making a training dataset of these new emotion categories will be easily compensated by the finer details offered by this new coding scheme.

It is important now to consider what lies beyond the attractive simplicity and convenience of the basic-emotions assumption, Barrett (2017) have presented an alternative for this view in her book How emotions are made by combining the most recent advances in neuroscience and cognitive psychology. She terms her theory in the theory of constructed emotion. This theory states that emotions need to be treated as abstract categories, which are the mental representations of our bodily states to direct our movements while optimizing the use of our metabolic resources. The direct implication of this view is that emotions cannot readily be told apart by only considering our physiological responses, facial expressions, voices, etc. To accurately detect emotions, we need information about the higher abstractions relevant to the contexts such emotions are formed. For example, emotion like pride or honor can be construed differently across culture (Han, 2016; Frey, Onyewuenyi, Hymel, Gill, \& Pearson,2020). 
Given the context of globalization and hyperconnectivity of Web 2.0; it is important to consider that the implications of the process of cultural evolution (Vuongt et al., 2019), cultural additivity (Vuong et al., 2018, 2020), and acculturation (Vuong \& Napier, 2015) for the development of emotional AI and empathic media. If we start with the premise of the Ekman's "basic emotions," these processes of cultural transformation dictate that the accuracy of our AI systems will depend on constantly getting the most updated data about how humans infer emotions. If we start with the premise of Barrett (2017)'s theory of constructed emotion, the cultural processes mentioned above imply the input higher-abstraction model of acculturation of emotions are necessary to make correct inference.

Two of the major ethical concerns in McStay's book are the loss of privacy and autonomy. McStay proposes a set of dignifying norms for all stakeholders to work with data about emotions. This set of norms are derived from "the liberal principles of autonomy, consent, control, empowerment, freedom, transparency, and trust" (p.188). Yet, these concerns should be considered together with different modes of empathic media derived from two alternative theories of emotions.

(Words count: 985)

\section{References}

Barrett, L. F. (2017). How emotions are made: The secret life of the brain. London: Houghton Mifflin Harcourt.

Ekman, P. (1999). Basic Emotions. In T. Dalgleish \& M. Power (Eds.), Handbook of Cognition and Emotion. Sussex, UK: John Wiley \& Sons. 
Ekman, P., Levenson, R. W., \& Friesen, W. V. (1983). Autonomic nervous system activity distinguishes among emotions. Science, 221(4616), 1208. doi:10.1126/science.6612338

Frey, K. S., Onyewuenyi, A. C., Hymel, S., Gill, R., \& Pearson, C. R. (2020). Honor, face, and dignity norm endorsement among diverse North American adolescents: Development of a Social Norms Survey. International Journal of Behavioral Development, 0165025420949690. doi:10.1177/0165025420949690

Han, K.-H. (2016). The feeling of "face" in Confucian society: From a perspective of psychosocial equilibrium. Frontiers in psychology, 7, 1055.

Ho, T. M. (2020, October 22). Beyond empathic media. OSF Preprints. Retrieved from osf.io/avjpk

McStay, A. (2018). Emotional AI: The rise of empathic media. London: Sage.

Vuong, Q. H. (2020). Reform retractions to make them more transparent. Nature, 582(7811), 149.

Vuong, Q. H., \& Napier, N. K. (2015). Acculturation and global mindsponge: an emerging market perspective. International Journal of Intercultural Relations, 49, 354-367.

Vuong, Q.-H., Bui, Q.-K., La, V.-P., Vuong, T.-T., et al. (2019). Cultural evolution in Vietnam's early 20th century: A Bayesian networks analysis of Hanoi Franco-Chinese house designs. Social Sciences \& Humanities Open, $1(1), \quad 100001$. doi:https://doi.org/10.1016/j.ssaho.2019.100001

Vuong, Q.-H., Ho, M.-T., Nguyen, H.-K. T., et al. (2020). On how religions could accidentally incite lies and violence: folktales as a cultural transmitter. Palgrave Communications, 6(1), 82. 
